\title{
Safety profile of anti-tumor necrosis factor therapy in inflammatory bowel disease - a single center experience
}

\author{
Piotr Eder, Liliana Łykowska-Szuber, Iwona Krela-Kaźmierczak, Kamila Stawczyk-Eder, \\ Katarzyna Klimczak, Aleksandra Szymczak, Krzysztof Linke
}

Department of Gastroenterology, Human Nutrition and Internal Diseases, Poznan University of Medical Sciences Heliodor Święcicki Clinical Hospital in Poznan, Poland

\begin{abstract}
Introduction. The data on the safety profile of anti-tumor necrosis factor (anti-TNF) therapy in real-life patients cohorts with inflammatory bowel disease (IBD) still are lacking.

Aim. The aim of the study was to assess the adverse events (AE) of anti-TNF therapy in a single Polish IBD center.

Material and methods. Data on the safety of anti-TNF therapy among Crohn's disease (CD) and ulcerative colitis (UC) patients treated in years 2009-2015 at the Department of Gastroenterology, Human Nutrition and Internal Diseases of Poznań University of Medical Sciences were analyzed.

Results. There were 41 AE/188 therapies reported (21.8\%) - 39/176 (22.1\%) in CD and 2/12 (16.6\%) in UC patients. The most common AE were infections - 10.1\%, followed by dermatological complications $-2.6 \%$, and infusion allergic reactions $-2.1 \%$. The majority of AE (27/41 - 66\%) were treated successfully or resolved without a treatment. The frequency of $A E$ among patients treated with different molecules was similar - 17/85 $(20 \%)$ in the adalimumab group, $20 / 91(21.9 \%)$ in the originator infliximab group, and $4 / 18(22.2 \%)$ in the biosimilar infliximab group. Concomitant treatment seemed not to influence the AE risk.

Conclusions. Anti-TNF therapy, which is used among the most severely ill IBD patients, seems to be safe. However, further assessment is needed in real-life patients cohorts, especially to assess the long-term safety of anti-TNF treatment in IBD.
\end{abstract}

Keywords: adalimumab, adverse events, Crohn's disease, infliximab, ulcerative colitis.

\section{Introduction}

Anti-tumor necrosis factor alpha (anti-TNF) therapy has significantly improved therapeutic possibilities in inflammatory bowel disease (IBD) in the last decade. High clinical efficacy of this novel therapy results in more and more common application of anti-TNF agents both in Crohn's disease (CD), and in ulcerative colitis (UC) [1]. Also in Poland, the number of anti-TNF-treated patients increases each year. Infliximab (IFX) and adalimumab (ADA) are the two most frequently used monoclonal antibodies neutralizing TNF-alpha in every- day clinical practice. Additionally, since 2014 new biosimilar forms of IFX are also used in Poland next to the originator IFX [2]. Since the introduction of biosimilars has decreased the costs of biological therapy, the treatment is becoming even more accessible.

One of the most important issues connected with each new therapy are safety profile and drug-related adverse events (AE). Anti-TNF therapy is generally considered to be safe, however, there are still many unanswered questions regarding, for example, the influence of IFX or ADA on the risk of infectious disease or malignancies [3]. 
The vast majority of data in terms of the safety profile of anti-TNF antibodies come from clinical trials. This has, however, significant limitations, as the populations included in clinical trials are strictly selected. Thus, the homogenous structure of the study groups in the trials does not reflect the heterogeneity and complexity of the clinical course of CD and UC. Moreover, each year new, unexpected and rare AE of anti-TNF treatment are reported. That is why we still need real-life data on the $A E$ related to anti-TNF therapy in IBD. In the current study, we present our experience and data on the safety profile of anti-TNF treatment in IBD patients, treated in our center in years 2009-2015.

\section{Material and methods}

A retrospective analysis of the safety profile of anti-TNF therapy applied among IBD patients treated at the Department of Gastroenterology, Human Nutrition and Internal Diseases of Poznań University of Medical Sciences was performed. All AE reported from January 2009 till May 2015 were collected.

The AE was defined as each unexpected medical event occurring during or after stopping the anti-TNF therapy which might be connected with the treatment. The AE were divided into those which were undoubtedly related to anti-TNF therapy and in which relation to the treatment was questionable, but theoretically possible. We also divided them into AE occurring during the therapy and after stopping it.
Serious adverse event (SAE) was defined as each $A E$ which significantly influenced the course of the therapy, resulting in death, a need for surgery or a need for the change in the concomitant pharmacological therapy, or a need for hospitalization.

Additionally, clinical data and laboratory results were collected.

An informed consent approved by the Institutional Review Board at the Heliodor Święcicki Clinical Hospital was obtained from each participant of the study.

Data were presented as means with standard deviations (SD). Categorized data were assessed with the Fisher exact test. A p value $<0.05$ was considered significant. All data were analyzed using the GraphPad Prism Version 6.0 (GraphPad Software Inc., USA).

\section{Results}

12 UC and 134 CD patients were treated with anti-TNF antibodies between January 2009 and May 2015. Considering that, in the case of CD - 101 patients were treated once, 26 - twice, 5 patients - three times and 2 patients - four times, the whole number of inclusions to the biological therapy program was 188 (94 women and 94 men). The baseline characteristics of the analyzed group is presented in Table 1 .

ADA was introduced 79 times, the originator IFX - 86 times and the biosimilar IFX 17 times. Additionally, in further 5 cases there was a need of changing the drug from the originator IFX to ADA and in 1 case

Table 1. Baseline characteristics of the whole study group $(n=188)$. Data are presented as means with standard deviations

\begin{tabular}{|c|c|c|}
\hline Feature & $\begin{array}{l}\text { Crohn's disease } \\
(n=176)\end{array}$ & $\begin{array}{l}\text { Ulcerative colitis } \\
\qquad(n=12)\end{array}$ \\
\hline Age (years) & $34 \pm 12$ & $32 \pm 12$ \\
\hline Male/female - $n$ & $87 / 89$ & $7 / 5$ \\
\hline Disease duration (years) & $5 \pm 4$ & $4 \pm 3$ \\
\hline C-reactive protein (mg/l) & $26.6 \pm 31.7$ & $42.7 \pm 31.6$ \\
\hline Erythrocyte sedimentation rate $(\mathrm{mm} / \mathrm{h})$ & $30 \pm 21$ & $43 \pm 32$ \\
\hline Hemoglobin ( $\mathrm{g} / \mathrm{dl})$ & $13.1 \pm 6.1$ & $11.6 \pm 2.4$ \\
\hline White blood cell count $\left(10^{3} / \mathrm{mm}^{3}\right)$ & $7.8 \pm 3.3$ & $11.1 \pm 3.1$ \\
\hline Platelets $\left(10^{3} / \mathrm{mm}^{3}\right)$ & $379 \pm 130$ & $363 \pm 117$ \\
\hline Disease location - \% & $\begin{array}{c}\text { L1 (ileal) }-38 \% \\
\text { L2 (colonic) }-20 \% \\
\text { L3 (ileocolonic) }-42 \%\end{array}$ & $\begin{array}{l}\text { E2 (left-sided) - 8\% } \\
\text { E3 (pancolitis) - 92\% }\end{array}$ \\
\hline \multicolumn{3}{|l|}{ Medications - \% } \\
\hline Steroids & $44 \%$ & $100 \%$ \\
\hline Azathioprine & $61 \%$ & $66 \%$ \\
\hline Aminosalicylates & $90 \%$ & $92 \%$ \\
\hline Antibiotics & $37 \%$ & $100 \%$ \\
\hline
\end{tabular}


- from the biosimilar IFX to ADA, because of the injection allergic reaction.

There were $41 \mathrm{AE} / 188$ therapies noted (frequency - 21.8\%) - 39/176 (22.1\%) in CD and 2/12 (16.6\%) in UC patients. $25 / 41$ (60.9\%) were assessed as SAE [23/39 (58.9\%) in CD, and 2/2 (100\%) in UC patients]. In 7 cases the AE were undoubtedly related to anti-TNF therapy (4 cases of allergic infusion reaction, 2 cases of drug-induced psoriasis in the same patient, 1 case of skin abscess at a drug injection place). In 34 cases the association with anti-TNF treatment was only hypothetical and more or less probable. In 2 cases (colon lymphoma, perineal cancer) the adverse event occurred > 12 months after finishing the therapy, in 39 cases during the therapy.

The characteristics of all $A E$ in the study group is presented in Table 2.

$27 \mathrm{AE}$ were treated successfully or resolved without a treatment, in 8 cases - the therapy was not successful (4 recurrent skin infections, 2 cases of resistant drug-induced psoriasis, 1 case of recurrent Clostridium difficile infection, and 1 death because of cancer of perineal region). 5 patients are still under treatment because of the AE possibly related to anti-TNF therapy, in 1 case we have no data on the further course of the AE.

The frequency of $A E$ among patients treated with different molecules was similar - 17/85 (20\%) in the ADA exposed group, 20/91 (21.9\%) in the originator IFX exposed patients, and 4/18 (22.2\%) in the biosimilar IFX exposed group.
There were no statistically significant differences in the frequency of $\mathrm{AE}$ regarding the concomitant treatment with azathioprine (34\% without azathioprine vs. $19 \%$ with azathioprine; $p=0.07)$, concomitant treatment with steroids (19\% without steroids vs. $23.3 \%$ with steroids; $p=0.5$ ). Patients who were treated for the second, third or fourth time were not at risk of developing more commonly AE when compared with those treated only once ( $26 \%$ vs. $20 \%$, respectively; $p=0.5)$. The frequency of AE was higher among women, however, without statistical significance (24\% vs. $19 \%$, respectively; $p=0.5$ ).

\section{Discussion}

Well balanced safety profile is one of the most important elements influencing the acceptance of every therapy. There are no medicines, however, which would not be associated with a risk of developing AE. The safety profile of anti-TNF antibodies seems to be acceptable, especially when considered their relevantly high clinical efficacy in untreatable conditions such as $\operatorname{IBD}[3,4]$. Nevertheless, there are still very few data in that subject coming from real-life patients populations. Moreover, there are no data describing this problem among adult IBD patients in Poland, and only one paper concerning Polish pediatric patients [5].

The overall rate of $A E$ in our study cohort was $22 \%$. This rate seems not to be high, when considered the qualification criteria for anti-TNF therapy in Poland.

Table 2. Characteristics of adverse events in patients with inflammatory bowel disease treated with anti-tumor necrosis factor therapy

\begin{tabular}{|c|c|c|c|}
\hline & Crohn's disease $(n=176)$ & Ulcerative colitis $(n=12)$ & All patients $(n=188)$ \\
\hline Infections & 18 & 1 & $19(10.1 \%)$ \\
\hline Perianal abscess & 6 & 0 & 6 \\
\hline Upper respiratory tract infections & 3 & 0 & 3 \\
\hline Skin infections & 8 & 0 & 8 \\
\hline Gastrointestinal infections & 0 & 1 & 1 \\
\hline Other infections & $\begin{array}{l}1 \text { case of varicella } \\
1 \text { intraabdominal abscess }\end{array}$ & 0 & 2 \\
\hline Dermatological adverse events & $\begin{array}{l}2 \text { cases of phototoxic reactions } \\
2 \text { cases of drug-induced psoriasis } \\
1 \text { case of dry skin syndrome }\end{array}$ & 0 & $5(2.6 \%)$ \\
\hline Hematological adverse events & $\begin{array}{l}2 \text { cases of leucopenia } \\
1 \text { case of thrombocytopenia }\end{array}$ & 0 & $3(1.5 \%)$ \\
\hline Malignancies & $\begin{array}{l}1 \text { case of perineal cancer } \\
1 \text { case of non-Hodgkin lymphoma }\end{array}$ & 1 case of brain tumor & $3(1.5 \%)$ \\
\hline Infusion allergic reactions & 4 & 0 & $4(2.1 \%)$ \\
\hline Other adverse events & $\begin{array}{l}1 \text { case of nausea } \\
1 \text { case of arthralgia } \\
2 \text { cases of liver enzymes elevation } \\
2 \text { cases of ileus }\end{array}$ & 0 & $6(3.1 \%)$ \\
\hline
\end{tabular}


Namely, we are using the "step-up" approach, in which only the most severely ill patients, not responding to all other classical drugs, can receive anti-TNF antibodies $[6,7]$.

It was shown, that the most frequent $\mathrm{AE}$ of anti-TNF therapy were infectious complications, which were noted among $10.1 \%$ of patients. This is in accordance with previous data [4]. For example, in a retrospective study on 500 patients treated with IFX in the Mayo Clinic, the frequency of infections was $8.2 \%[4,8]$. In a study by Lees et al., which included 620 patient-years of follow-up, the incidence of infections was 9.9\% [4, 9]. The authors showed, that the majority of infectious AE occurred among patients receiving concomitant immunosuppressive drugs. In our patients' cohort we did not observe an increase of infections rate among patients on combo therapy. However, it has to be noted that the vast majority of patients were on steroids and/or azathioprine while starting anti-TNF treatment, so it is hard to definitively assess, whether this fact influenced the infectious complication risk. There were no cases of reactivated tuberculosis, which seems to be one of the most serious infectious AE in the course of anti-TNF therapy [10]. This shows that currently used algorithm for the exclusion of latent tuberculosis in Poland, using chest X-ray and skin tuberculin tests or interferon-gamma release assays (IGRA) before starting the treatment is effective.

One of the most frequent infectious complication in our study group were perianal abscesses (6 cases). This clinical situation could be also interpreted as a manifestation of $C D$, however, it seems that the link between anti-TNF therapy and the formation of perianal abscess is strong enough to classify it as AE [11]. This complication in all cases took place in the early phase of treatment and, hypothetically, was a result of closure of external skin opening of fistulas. This, in turn, prevented evacuation of infected content of the fistula tract which led to the collection of pus and formation of perianal abscess. Since this is a severe complication, we changed the therapeutic approach in the cases of complex perianal $C D$, performing parallel aggressive surgical treatment, including negative wound pressure therapy. As a result, since 2013, there has been only one case of perianal abscess. This example shows that the optimization and individualization of anti-TNF therapy can improve therapeutic outcomes and decrease the risk of $\mathrm{AE}$.

Interestingly, we noted also several cases of dermatological AE in our series. This number could be even greater if injection site reactions or skin infections were classified to this subgroup of AE. This is in accordance with the current knowledge in terms of the safety of anti-TNF agents $[12,13]$. It seems that dermatological effects of anti-TNF treatment were underestimated. Rheumatologic data show that even up to $25 \%$ of patients on anti-TNF drugs had dermatological complications, and this was also confirmed in IBD patients [13, 14]. The most clinically significant dermatological $A E$ seem to be skin malignancies. There are, however, conflicting data in terms of the influence of anti-TNF therapy on the risk of non-melanoma skin cancer (NMSC) or skin melanoma [13]. Moreover, in a study performed by Long et al. in a cohort of 108579 IBD patients it was shown that the disease by itself can be associated with higher risk of skin melanoma and NMSC, especially in individuals with CD [15]. Additionally, immunosuppressive drugs, like azathioprine and methotrexate, can also influence the malignancy rates [13].

In our study cohort there was no case of skin malignancy, but we noted another interesting dermatological $A E$ - drug-induced psoriasis. This was observed twice in the same CD patient (the first time - during the course of maintenance IFX treatment, and then three years later - as a result of ADA therapy). The etiology of this immune-mediated complication is unknown [13]. It is believed that anti-TNF agents, being anti-inflammatory molecules, in some proportion of patients can cause paradoxical pro-inflammatory reactions, which is hypothetically mediated by a local uncontrolled production of interferon- $\alpha$, resulting from TNF blockade $[13,16]$. Current data show, that this phenomenon is not rare. In one of the most recent reviews of this subject, Denadai et al. reported that the highest risk of developing anti-TNF-induced psoriasis is among CD patients on IFX [17]. Skin lesions can appear at any moment of the therapy, but the most frequent time is between third and fourth IFX infusion [13].

We reported 3 cases of malignancies in our study group. In the case of two of them, however, the link between anti-TNF therapy was very weak. In the case of a patient with perineal cancer, the main etiological factor was a severe, long-lasting perianal CD, with multiple perianal and vaginal fistulas. In the second one - brain tumor was diagnosed just after first dose of IFX in a patient with fulminant UC, so the association with anti-TNF therapy is very unlikely. We also reported a case of non-Hodgkin lymphoma (NHL) of the colon. This patient was treated with ADA, however, the patient was also treated with experimental drug in clinical trials in the past because of a very severe CD course. That is why, it is also difficult to assess what was the main etiological factor of NHL in this case - anti-TNF thera- 
py, concomitant and past treatment, or active, severe and long-lasting disease by itself. The literature review also brings conflicting data in terms of the association between anti-TNF therapy and lymphoma risk [10, 18, 19]. Recent meta-analyses show that the risk of developing lymphoma is slightly increased in patients with IBD and it is related to the use of immunosuppressive drugs or both - immunosuppressants together with anti-TNF agents [18].

Our study has several limitations. The main one is the retrospective nature of the analysis, as it theoretically could lead to underestimation of the $A E$ rate. However, as it was also noted in another retrospective study on the safety of anti-TNF therapy among Polish pediatric IBD patients, all patients included in the analysis were treated according to the guidelines of Polish National Health Fund, and we are obliged to strictly monitor the course of the therapy [5]. As a result, all data are registered and collected, thus they seem to be reliable enough to perform the analysis. Another limitation is the definition of $A E$, as it may influence the rate of reported complications of the therapy. Some of the were $A E$ reported, however, their association with anti-TNF therapy seemed to be very poor. On the other hand, we excluded some clinical situations (for example anemia), recognizing them as disease manifestation, not therapeutic complications. Nevertheless, it should be mentioned, that there is no commonly accepted definition of $A E$, and all data in this subject should be interpreted with respect to specific definition used in a particular, analyzed study.

\section{Conclusions}

To conclude, in the current study safety profile of anti-TNF therapy in IBD real-life patients cohort is presented. It was shown for the first time among Polish adult IBD patients, that the therapy is generally well-tolerated and the majority of the AE disappear after stopping the treatment and/or introducing a specific therapy. We did not show any significant differences in drug-related complication rate either between different molecules or between the originator and biosimilar IFX, however, the number of patients treated with biosimilars is still small. Proper qualification to the therapy, strict monitoring and optimizing the treatment can decrease or eliminate the risk of several $A E$. However, since the observation time of the safety profile of anti-TNF treatment is still short (10-15 years), further assessment is needed in this respect, especially in terms of long-term therapy of IBD.
Acknowledgements

We would like to thank Agnieszka Kurek for her writing assistance.

\section{Conflict of interest statement}

The authors declare that there is no conflict of interest in the authorship or publication of contribution.

\section{Funding sources}

P. Eder, L. Łykowska-Szuber, K. Klimczak received lecture fees from Abbvie Poland.

\section{References}

1. Amiot A, Peyri-Biroulet L. Current, new and future biological agents on the horizon for the treatment of inflammatory bowel diseases. Therap Adv Gastroenterol. 2015 Mar;8(2):66-82.

2. Gomollon F. Biosimilars in inflammatory bowel disease: ready for prime time? Curr Opin Gastroenterol. 2015 Jul;31(4):290-295.

3. Sousa P, Allez M. Complications of biologics in inflammatory bowel disease. Curr Opin Gastroenterol. 2015 Jul;31(4):296-302.

4. Stallmach A, Hagel S, Bruns T. Adverse effects of biologics used for treating IBD. Best Pract Res Clin Gastroenterol. 2010 Apr;24(2):167-182.

5. Szymańska E, Dądalski M, Oracz G, Kierkuś J. Safety profile of anti-TNF agents in Polish pediatric patients with Crohn's disease. Austin J Gastroenterol. 2014 Aug;1(4): 1016.

6. Łodyga M, Eder P, Bartnik W, Gonciarz M, Kłopocka M, Linke $\mathrm{K}$ et al. Guidelines for the management of Crohn's disease. Recommendations of the Working Group of the Polish National Consultant in gastroenterology and the Polish Society of Gastroenterology. Prz Gastroenterol 2012 Dec;7(6):317-338.

7. Eder $P$, Łodyga $M$, Łykowska-Szuber $L$, Bartnik W, Durlik M, Gonciarz $M$ et al. Guidelines for the management of ulcerative colitis. Recommendations of the Working Group of the Polish National Consultant in gastroenterology and the Polish Society of Gastroenterology. Prz Gastroenterol 2013 Jan;8(1):1-20.

8. Colombel JF, Loftus EV, Tremaine WJ, Egan EJ, Harmsen WS, Schleck CD et al. The safety profile of infliximab in patients with Crohn's disease: the Mayo Clinic experience in 500 patients. Gastroenterology. 2004 Jan;126(1): 19-31.

9. Lees CW, Ali Al, Thompson Al, Ho GT, Forsythe RO, Marquez $L$ et al. The safety profile of anti-tumour necrosis factor therapy in inflammatory bowel disease in clinical practice: analysis of 620 patient-years follow-up. Aliment Pharmacol Ther. 2009 Feb;29(3):286-297.

10. Targownik LE, Bernstein CN. Infectious and malignant complications of TNF inhibitor therapy in IBD. Am J Gastroenterol. 2013 Dec;108(12):1835-1842.

11. Gecse K, Khanna R, Stoker J, Jenkins JT, Gabe S, Hahnloser D et al. Fistulizing Crohn's disease: Diagnosis and management. United European Gastroenterol J. 2013 Jun;1(3):206-213.

12. Freling E, Baumann C, Cuny JF, Bigard MA, Schmutz JL, Barbaud $A$ et al. Cumulative incidence of, risk factors for, and outcome of dermatological complications of anti- 
-TNF therapy in inflammatory bowel disease: a 14-year experience. Am J Gastroenterol. 2015 Aug;110(8):11861196.

13. Mocci G, Marzo M, Papa A, Armuzzi A, Guidi L. Dermatological adverse reactions during anti-TNF treatments: focus on inflammatory bowel disease. J Crohns Colitis. 2013 Nov;7(10):769-779.

14. Lee HH, Song IH, Friedrich M, Gauliard A, Detert J, Rowert J et al. Cutaneous side-effects in patients with rheumatic diseases during application of tumour necrosis factor-alpha antagonists. $\mathrm{Br} J$ Dermatol. 2007 Mar;156(3):486-491.

15. Long MD, Martin CF, Pipkin CA, Herfarth HH, Sandler RS, Kappelman MD. Risk of melanoma and nonmelanoma skin cancer among patients with inflammatory bowel disease. Gastroenterology. 2012 Aug;143(2):390-399.

16. Cullen G, Kroshinsky D, Cheifetz AS, Korzenik JR. Psoriasis associated with anti-tumour necrosis factor therapy in inflammatory bowel disease: a new series and a review of 120 cases from the literature. Aliment Pharmacol Ther. 2011 Dec;34(11-12):1318-1327.

17. Denadai R, Teixera FV, Saad-Hossne R. The onset of psoriasis during the treatment of inflammatory bowel diseases with infliximab: should biological therapy be suspended? Arq Gastroenterol. 2012 Apr-Jun;49(2):172-176.

18. Lakatos PL, Miheller P. Is there an increased risk of lymphoma and malignancies under anti-TNF therapy in IBD? Curr Drug targets. 2010 Feb;11(2):179-186.
19. Kopylov U, Vutcovici M, Kezouh A, Seidman E, Bitton A, Afif W. Risk of lymphoma, colorectal and skin cancer in patients with IBD treated with immunomodulators and biologics: a Quebec claims database study. Inflamm Bowel Dis. 2015 Aug;21(8):1847-1853.

Acceptance for editing: 2015-02-10 Acceptance for publication: 2015-03-30

Correspondence address: Piotr Eder Department of Gastroenterology, Human Nutrition and Internal Diseases, Poznan University of Medical Sciences Heliodor Swiecicki Clinical Hospital 49 Przybyszewskiego Street, 60-355 Poznan, Poland phone: +48618691343 fax: +48 618691686 email: piotr.eder@op.pl 